\title{
INFLUÊNCIA DA ALIMENTAÇÃO NO POTENCIAL REPRODUTIVO DE CRISOPÍDEOS
}

\section{Luciano José Ribeiro ${ }^{1}$ Sérgio de Freitas ${ }^{1}$}

\section{RESUMO}

Avalinu-se a influência da alimentação dos adultos no potencial reprodutivo de Chrysoperla externa (Hagen, 1861). As larvas foram criadas em grupos, em potes plásticos em condições de laboratório $\left(25 \pm 2^{\circ} \mathrm{C}, 75 \pm 10 \%\right.$ UR e 10 horas de fotofase), alimentadas apenas com ovos de Sitotroga cerealella (Olivier). As larvas foram alimentadas até o início do encasulamento. Após a emergência, os adultos, sexados e acasalados, foram mantidos em gaiolas com as dietas. $\mathrm{O}$ experimento contou com cinco dietas, com 20 repetições para cada uma. Foram avaliados os seguintes parâmetros: períodos de pré-oviposição, oviposição e pós-oviposição; oviposição diária e total por fêmea e longevidade dos adultos. Os resultados indicam que a alimentação fornecida na fase adulta dos insetos é responsável por alterações no desenvolvimento e no seu potencial reprodutivo e que a dieta à base de mel, levedura de cerveja e pólen levou aos melhores resultados.

Palavras-chave: crisopídeos, predador, adulto, dieta, oviposição.

1 Dep. de Entomologia e Nematologia, FCAV-UNESP, Câmpus de Jaboticabal. Rodovia Carlos Tonanni, Km 5, 14870-000, Jaboticabal, SP, Brasil. 


\section{ABSTRACT}

\section{INFLUENCE OF FOOD ON Chrysoperla externa (HAGEN) (NEUROPTERA: CHRYSOPIDAE) REPRODUCTIVE POTENTIAL}

The influence of the adult food in the reproductive potential of Chrysoperla externa (Hagen, 1861) (Neuroptera: Chrysopidae) was evaluated. The larvae groups were reared in plastic boxes in laboratory conditions $\left(25 \pm 2^{\circ} \mathrm{C}, 75 \pm 10 \% \mathrm{RH}\right.$ and 10 -hour photophase), and fed with Sitotroga cerealella (Olivier) eggs until the beginning of the pupal stage. After emergence, the adults were sexed and coupled, maintained in cages, and fed with artificial diet. The following parameters were evaluated: preoviposition, oviposition and postoviposition periods; daily and total oviposition per female and adult longevity. The results have indicated that adult food is important to reproductive potential and development changes. The best results were obtained with adults fed with a diet based on honey, yeast and pollen.

Key words: green lacewing, predator, adult, diet, oviposition.

\section{INTRODUÇÃO}

Os crisopídios, neurópteros da família Chrysopidae, são consid.rados inimigos naturais chave em vários programas de Manejo Integrado de Pragas (MIP). Atuam como agentes de controle biológico em diferentes culturas anuais, perenes e casas de vegetação (New, 1975; Ridgway \& Murphy, 1984). As presas dos crisopídeos geralmente são insetos que apresentam cutícula fina e facilmente perfurável por sua mandíbula, bem como presas relativamente pequenas, lentas ou sésseis. Tais características conferem um amplo espectro de alimentação aos crisopídeos, sendo sua dieta composta por pulgões, cochonilhas, moscas-brancas, ovos e lagartas de lepidópteros, ácaros e pequenas aranhas (Ridgway \& Kinzer, 
1974; New, 1974; Canard \& Duelli, 1984; Freitas \& Fernandes, 1996). Segundo Nuñez (1988), Chrysoperla externa (Hagen) é uma espécie bastante promissora para programas de Controle Biológico de Pragas. Suas principais características são a ação predatória durante a fase larval, ampla distribuição geográfica, presença de adultos em cultivos durante todo o ano, fácil criação em cativeiro, potencial de adaptação a diferentes ambientes e sua resistência a vários inseticidas. Freitas \& Fernandes (1996) ressaltaram, além da voracidade, sua grande plasticidade ecológica, sendo encontrados em diferentes agroecossistemas associados a diferentes pragas.

A alimentação fornecida aos adultos de crisopídeos é um dos principais fatores que regem o sucesso da criação massal do predador. Dietas nutricionalmente inadequadas podem interferir em diferentes aspectos da biologia destes insetos, entre eles, a fecundidade, o crescimento, o desenvolvimento e a longevidade (Hagen, 1950, Cañedo \& Lizárraga, 1988; Parra, 1994; Carvalho et al., 1997). Segundo Parra (1994), para o crescimento, desenvolvimento e produção de ovos, os aminoácidos são componentes essenciais, sendo trazidos da alimentação larval, ou tendo de ser providos na fase adulta.

O presente trabalho teve como objetivo avaliar a influência da alimentação fornecida a C. externa na fase adulta, no seu desenvolvimento e potencial reprodutivo.

\section{MATERIAL E MÉTODOS}

O experimento foi conduzido no Laboratório de Criação Massal de Crisopídeos do Departamento de Entomologia e Nematologia da Faculdade de Ciências Agrárias e Veterinárias, FCAV/UNESP, Jaboticabal, $\mathrm{SP}$, em condições controladas de temperatura $\left(25 \pm 2^{\circ} \mathrm{C}\right)$, umidade relativa do ar $(75 \pm 10 \%)$, e fotoperíodo (10 horas de fotofase).

Utilizou-se a geração $\mathrm{F}_{1}$ obtida de adultos coletados no campo, em pomares de citros, de noz-macadâmia, de goiaba e de pínus, nos me- 
ses de março e abril de 1997. Os adultos foram levados ao Laboratório de Biossistemática de Crisopídeos (FCAV/UNESP) para identificação e sexagem.

Os insetos foram mantidos em gaiolas de PVC, descritas por Ferreira (1996), com algumas adaptações para o provimento de água e alimento. Duas vezes por semana foram alimentados com dieta à base de mel e levedura de cerveja (2:1). O alimento, oferecido aos adultos em um pedaço de espuma de borracha, utilizado para tampar o frasco com água destilada, foi então vertido sobre uma abertura no tecido que cobria a gaiola. O reabastecimento dos frascos com água e a limpeza das espumas, foram feitos uma vez por semana. Para criação estoque formaram-se 6 gaiolas com 10 casais em cada uma.

Ovos de até um dia foram recolhidos e mantidos em potes plásticos. Após eclosão, as larvas foram alimentadas com ovos de Sitotroga cerealella até o início do encasulamento. Com a emergência dos adultos, 100 casais foram separados em gaiolas e receberam durante seu desenvolvimento uma das dietas testadas (Tabela 1), conforme metodologia já apresentada. Cada tratamento contou com 20 gaiolas, com 1 casal cada uma.

As observações foram feitas diariamente em todas as gaiolas. Foram avaliados os seguintes parâmetros: períodos de pré-oviposição, oviposição e pós-oviposição; número de ovos/fêmea/dia; número total de ovos por fêmea e longevidade dos adultos machos e fềmeas.

Os dados foram transformados e submetidos a análise de variância, sendo as médias comparadas pelo teste de Tukey a 5\% de probabilidade.

\section{RESULTADOS E DISCUSSÃO}

A variação na composição das dietas fornecidas às larvas de Chrysoperla externa levaram a algumas diferenças significativas nas características avaliadas no experimento (Tabelas 2,3 e 4). 
Tabela 1. Composição das dietas fornecidas aos adultos da geração $\mathrm{F}_{1}$ de Chrysoperla externa (Hagen), Jaboticabal, SP.

\begin{tabular}{|c|c|}
\hline Dieta & Composição \\
\hline $\mathrm{ML}$ & Mel + Levedura de Cerveja (2:1) \\
\hline MLP & Mel + Levedura de Cerveja $(2: 1)+$ pólen $(10 \%)$ \\
\hline MLD3 & Mel + Levedura de Cerveja (2:1) + solução de diamônio fosfato a 3\% (10\%) \\
\hline MLD8 & Mel + Levedura de Cerveja (2:1) + solução de diamônio fosfato a $8 \%(10 \%)$ \\
\hline DE & $\begin{array}{l}\text { 15mi de leite condensado; } 1 \text { ovo; } 1 \text { gema de ovo; } 30 \mathrm{~g} \text { de mel; } 20 \mathrm{~g} \text { de frutose; } 30 \mathrm{~g} \text { de Levedura } \\
\text { de Cerveja; } 30 \mathrm{~g} \text { de germe de trigo e água destilada (Degrande, 1996) }\end{array}$ \\
\hline
\end{tabular}

De acordo com os resultados nota-se, no entanto, que a alimentação não interferiu no período anterior ao início da oviposição, nem no período após a oviposição, evidenciado pela ausência de diferenças significativas entre os valores dos períodos de pré-oviposição e pósoviposição (Tabela 2). Entretanto, o período efetivo de oviposição foi significativamente diferente em função da dieta recebida pelos adultos de C. externa. Fêmeas adultas que receberam a dieta ML, ovipositaram durante cerca de 81 diaș, enquanto que as alimentadas com MLD8, ovipositaram cerca de 52 dias, com uma diferença de aproximadamente 29 dias. Esta diferença está diretamente relacionada com a vida útil do inseto no laboratório, possibilitando melhor aproveitamento dos recursos investidos na criação (Tabela 2).

A capacidade diária de oviposição das fêmeas não foi alterada em função das diferentes dietas fornecidas aos adultos (Tabela 3). No entanto, o número total de ovos postos foi significativamente diferente. A dieta MLD8 possibilitou o menor número médio de ovos postos por fềmea, cerca de 680, enquanto MLP, o maior, ficou em torno de 1060 ovos. Este fato está intimamente relacionado ao maior período efetivo de oviposição determinado por esta dieta. A superioridade da dieta que contém pólen 
Tabela 2. Período de pré-oviposição, oviposição e pós-oviposição de adultos da geração $\mathrm{F}_{1}$ de Chrysoperla externa (Hagen) que receberam diferentes dietas alimentares, Jaboticabal, SP. $\mathrm{EP}=$ erro padrão da média.

Duração do periodo (dias) ( $\pm \mathrm{EP})^{1}$

Dieta

(n)

\begin{tabular}{|c|c|c|c|c|}
\hline & & Pré-oviposiçäo ${ }^{2}$ & Oviposiçăo $0^{3}$ & Pós-owiposiçāa $0^{4}$ \\
\hline & ML & $\begin{array}{c}6,10 \pm 0,34 a \\
\{19)\end{array}$ & $\begin{array}{c}81,53 \pm 3,80 \mathrm{a} \\
(19)\end{array}$ & $\begin{array}{c}3,11 \pm 0,69 a \\
(79)\end{array}$ \\
\hline & $\mathrm{MLP}$ & $\begin{array}{c}5,28 \pm 0,39 \mathrm{a} \\
(18)\end{array}$ & $\begin{array}{c}79,22 \pm 6,81 \mathrm{ab} \\
(18)\end{array}$ & $\begin{array}{c}3,22 \pm 0,75 a \\
(78)\end{array}$ \\
\hline & MLD3 & $\begin{array}{c}4,80 \pm 0,16 a \\
(20)\end{array}$ & $\begin{array}{c}71,40 \pm 5,32 \mathrm{ac} \\
\text { (20) }\end{array}$ & $\begin{array}{l}3,25 \pm 0,94 a \\
(20)\end{array}$ \\
\hline & MLD 8 & $\begin{array}{c}5,32 \pm 0,46 \mathrm{a} \\
(19)\end{array}$ & $\begin{array}{c}52,84 \pm 6,01 \mathrm{c} \\
(19)\end{array}$ & $\begin{array}{c}2,47 \pm 0,8 a \\
(19)\end{array}$ \\
\hline & $\mathrm{DE}$ & $\begin{array}{c}5,39 \pm 0,27 a \\
(20)\end{array}$ & $\begin{array}{c}56,60 \pm 7,05 b c \\
(20)\end{array}$ & $\begin{array}{c}4,70 \pm 1,12 \mathrm{a} \\
(20)\end{array}$ \\
\hline F & & $2,43 n s$ & $4,82^{* *}$ & $0,77 \mathrm{~ns}$ \\
\hline $\mathrm{CV}$ & & $11,35 \%$ & $23,11 \%$ & $20,63 \%$ \\
\hline
\end{tabular}

'Médias seguidas de mesma letra, na mesma coluna, não diferem entre si pelo teste de Tukey a $5 \%$ de probabilidade.

2Para análise estatística os dados ( $\mathrm{x}$ ) foram transformados em $\sqrt{x+1}$.

${ }^{3}$ Para análise estatística os dados (x) foram transformados em $\sqrt{x}$.

${ }^{4}$ Para análise estatística os dados $(\mathrm{x})$ foram transformados em $\log (\mathrm{x}+5)$.

"nsão significativo a $5 \%$ de probabilidade.

**Significativo a $1 \%$ de probabilidade.

também foi verificada por Sundby (1967) ao avaliar a capacidade de oviposição de Chrysoperla carnea alimentada com dieta na presença e ausência deste componente. A superioridade provém de o pólen enriquecer a dieta com proteínas, que são fundamentais para a produção de ovos (Parra, 1994). Além disso, Panizzi \& Parra (1991) citam que em alguns tipos de pólen são encontrados mais de 14 carboidratos ligados a processos geradores de energia e síntese protéica nos insetos. 
Tabela 3. Capacidade média de oviposição diária e total de fêmeas da geração $\mathrm{F}_{1}$ de Chrysoperla externa (Hagen) que receberam diferentes dietas alimentares, Jaboticabal, SP. EP = erro padrão da média.

\begin{tabular}{|c|c|c|c|}
\hline & \multirow{2}{*}{ Dieta } & \multicolumn{2}{|c|}{$\begin{array}{l}\text { Ovipos ção (dias) (₫EP) } \\
(n)\end{array}$} \\
\hline & & Ovos/dia/fênea ${ }^{2}$ & Total de ovos/fêmea \\
\hline & $\mathrm{MI}$ & $\begin{array}{c}10,57 \pm 0,57 a \\
(19)\end{array}$ & $\begin{array}{c}935,89 \pm 53,41 a b \\
(19)\end{array}$ \\
\hline & MLP & $\begin{array}{c}13,11 \pm 0,86 \mathrm{a} \\
(18)\end{array}$ & $\begin{array}{c}1060,56 \pm 76,28 \mathrm{a} \\
(18)\end{array}$ \\
\hline & MLD3 & $\begin{array}{c}11,65 \pm 0,51 a \\
(20)\end{array}$ & $\begin{array}{c}918,65 \pm 64,82 a b \\
(20)\end{array}$ \\
\hline & MLD8 & $\begin{array}{c}11,01 \pm 1,80 \mathrm{a} \\
(19)\end{array}$ & $679,74 \pm 76,69 \mathrm{~b}$ \\
\hline & $\mathrm{DE}$ & $\begin{array}{c}12,70 \pm 0,63 a \\
(20)\end{array}$ & $\begin{array}{c}841,30 \pm 93,50 a b \\
(20)\end{array}$ \\
\hline E & & $1,70 \mathrm{~ns}$ & $3,38^{*}$ \\
\hline $\mathrm{Cl}$ & & $18,23 \%$ & $21,13 \%$ \\
\hline
\end{tabular}

'Médias seguidas de mesma letra, na mesma coluna, não diferem entre si pelo teste de Tukey a $5 \%$ de probabilidade.

${ }^{2}$ Para análise estatística os dados (x) foram transformados em $\sqrt{x}$ ${ }^{n}$ Não significativo a $5 \%$ de probabilidade.

*Significativo a $5 \%$ de probabilidade.

A utilização do diamônio fosfato na dieta como atrativo não interferiu significativamente no potencial de oviposição, não diferindo estatisticamente nas duas concentrações utilizadas, que por sua vez não diferiram da dieta ML.

A longevidade dos machos não foi influenciada pelas dietas fornecidas aos adultos, variando em torno de 57 a 80 dias (Tabela 4). Em relação às fêmeas, essa variação ficou, aproximadamente, entre 62 e 90 dias, com destaque para a dieta ML, que possibilitou a maior longevidade. 
Tabela 4. Longevidade média de adultos machos e fềmeas da geração $\mathrm{F}_{1}$ de Chrysoperla externa (Hagen) que receberam diferentes dietas alimentares. Jaboticabal, SP. EP = erro padrão da média.

\begin{tabular}{|c|c|c|c|}
\hline & \multirow{2}{*}{ Diela } & \multicolumn{2}{|c|}{$\begin{array}{l}\text { Longevidade (dias) }( \pm E P)^{1} \\
\qquad(n)\end{array}$} \\
\hline & & Macho ${ }^{2}$ & Fêmea ${ }^{2}$ \\
\hline & MIL & $\begin{array}{c}66,35 \pm 9,06 a \\
(20)\end{array}$ & $\begin{array}{l}90,21 \pm 3,96 \mathrm{a} \\
(79)\end{array}$ \\
\hline & $M \amalg P$ & $\begin{array}{l}80,95 \pm 7,66 \mathrm{a} \\
(20)\end{array}$ & $\begin{array}{c}87,89 \pm 6,88 \mathrm{ab} \\
(18)\end{array}$ \\
\hline & MLD3 & $\begin{array}{l}76,90 \pm 6,69 a \\
(20)\end{array}$ & $\begin{array}{l}82,95 \pm 6,10 a b \\
(20)\end{array}$ \\
\hline & MLD8 & $\begin{array}{l}78,45 \pm 8,61 \mathrm{a} \\
(20)\end{array}$ & $\begin{array}{l}62,68 \pm 5,75 \mathrm{~b} \\
(79)\end{array}$ \\
\hline & $\mathrm{DE}$ & $\begin{array}{l}57,45 \pm 5,59 \mathrm{a} \\
(20)\end{array}$ & $\begin{array}{l}67,15 \pm 7,61 \mathrm{~b} \\
(20)\end{array}$ \\
\hline $\mathrm{F}$ & & $1.53 \mathrm{~ns}$ & $4,20^{* *}$ \\
\hline $\mathrm{CV}$ & & $26,76 \%$ & $19,75 \%$ \\
\hline
\end{tabular}

${ }^{1}$ Médias seguidas de mesma letra, na mesma coluna, não diferem entre si pelo teste de Tukey a $5 \%$ de probabilidade.

${ }^{2}$ Para análise estatística os dados (x) foram transformados em $\sqrt{x}$

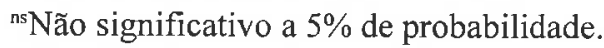

*Significativo a $5 \%$ de probabilidade.

Observa-se que, entre as dietas avaliadas, MLP apresentou tendência a melhores resultados, apesar de não diferir estatisticamente de ML. Acredita-se que a dieta à base de mel, levedura de cerveja e pólen seja a alternativa mais econômica e prática entre as dietas avaliadas para a criação massal do predador. 


\section{REFERÊNCIAS BIBLIOGRÁFICAS}

CANARD, M. S.; P. DUELLI, 1984. Predatory Behavior of Larvae and Cannibalism, p.92-9. In Canard, M., Y. Sémeria, T. R. New ' ed.) Biology of Chrysopidae. Haia: W. Junk, 323p.

CAÑEDO T. D. V.; T. A. LIZÁRRAGA, 1988. Dietas Artificiales para la Crianza en Laboratorio de Chrysoperla externa (Hagen) (Neuroptera: Chrysopidae). Rev. Peruana de Entomologia, 31: 83-5.

CARVALHO, C. F.; M. CANARD; C. ALAUZET, 1997. Variação Ponderal de Adultos de Chrysoperla mediterranea (Hölzel, 1972) (Neuroptera: Chrysopidae) em Função da Alimentação Imaginal. In: CONGRESSO BRASILEIRO DE ENTOMOLOGIA, 16, SALVADOR. Anais, p.70.

DEGRANDE, P.E. 1996. Otimização e Prática da Metodologia da IOBC para Avaliar o Efeito de Pesticidas Sobre Trichogramma cacoecidae (Trichogrammatidae) e Chrysoperla externa (Chrysopidae). Piracicaba, 109p. Tese (Doutorado em Entomologia) - Escola Superior de Agricultura Luiz de Queiroz, Universidade de São Paulo.

FERREIRA, R. J. 1996. Técnicas para Produção Massal de Crisopídeos (Neuroptera: Chrysopidae). Jaboticabal. 115p. Dissertação (Mestrado em Agronomia) - Faculdade de Ciências Agrárias e Veterinárias - Universidade Estadual Paulista.

FREITAS, S. de; O. A. FERNANDES 1996. Crisopídeos em Agroecossistemas. In: SIMPÓSIO DE CONTROLE BIOLÓGICO, 5, Foz do Iguaçu. Anais. p.283.

HAGEN, K. S. 1950 Fecundity of Chrysopa californica as Affected by Synthetic Foods. Jour. of Economic Entomology, 48:1001-4.

NEW, T. R. 1975. The biology of Chrysopidae and Hemerobiidae (Neuroptera), With Reference to Their Usage as Biocontrol Agents: a Review. Transactions of the Royal Entomology Society of London, 127: 115-40. 
NUÑEZ, Z. E. 1988. Ciclo Biológico y Crianza de Chrysoperla externa y Ceraeochrysa cincta (NEUROPTERA: CHRYSOPIDAE). Revista Peruana de Entomología, 31: 76-82.

PANNIZI, A. R.; PARRA, J. R. P. 1991. Ecologia Nutricional de Insetos e suas Implicações no Manejo de Pragas. São Paulo:Manole, 357p.

PARRA, J. R. P. 1994. Técnicas de Criação de Insetos para Programas de Controle Biológico. 2.ed. Piracicaba: ESALQ, 196p.

RIDGWAY, R. L.; R. E. KINZER, 1974. Chrysopids as Predators of Crop Pests. Entomophaga, 7: 45-51.

RIDGWAY, R. L.; W. L. MURPHY, 1984. Biological Control in the Field, p. 220-8. In: Canard, M., Y. Séméria, T. R. New (ed.) Biology of Chrysopidae. Haia, W. Junk, 323p.

SUNDBY, R. A. 1967. Influence of Food on the Fecundity of Chrysopa carnea (Stephens) (Neuroptera, Chrysopidae). Entomophaga, 12: $475-9$. 